\title{
Computational Modelling and Experimental Characterisation of Heterogeneous Materials
}

\author{
* A. J. Beveridge ${ }^{1}$, M. A. Wheel ${ }^{1}$, D. H. Nash $^{1}$ \\ ${ }^{1}$ Department of Mechanical Engineering \\ University of Strathclyde \\ Glasgow \\ UK, G1 1XJ \\ *andrew.beveridge@strath.ac.uk
}

\begin{abstract}
Heterogeneous materials can exhibit behaviour under load that cannot be described by classical continuum elasticity. Beams in bending can show a relative stiffening as the beam depth tends to zero, a size effect. Size effects are recognised in higher order continuum elastic theories such as micropolar elasticity. The drawback of higher order theories is the requirement of addition constitutive relations and associated properties that are often difficult to establish experimentally. Furthermore the finite element method, of great benefit in classical elasticity, has shown limitations when applied to micropolar elasticity. The determination of additional constitutive properties and the computational modelling of micropolar elasticity will be discussed in the context of a model heterogeneous material loaded in simple 3 point bending.

The model material was created by drilling holes in aluminium bar in a regular pattern, with the hole axis normal to the plane of bending. The bending tests show that a size effect is present. These results are compared against modelling the detailed beam geometries in the finite element package ANSYS, which again shows the size effect. These two bending test are used to extract the additional micropolar elastic material properties. A comparison is then made against analytical solutions, numerical solutions using a micropolar beam finite element and a micropolar plane stress control volume method.

It will be shown that the need for extensive experimental testing to determine the additional constitutive properties may not be necessary with the appropriate use of numerical methods.
\end{abstract}




\section{Introduction}

\section{Heterogeneous Materials}

A loaded material can be classed as either homogeneous; material behaviour is length scale independent, or heterogeneous; local variations in structure produce length scale dependence. This work describes the deformation of materials where the heterogeneous nature becomes significant. Classical elasticity is a continuum model for describing the deformation of homogeneous materials but it is insufficient when the scale of the local structure becomes significant. Therefore an approach differing from classical elasticity is required.

\section{Generalized Elastic Continuum Theories}

One approach is to use a model for a generalized elastic continuum, which is one that takes into account the detail of the underlying structure but is still a continuum model similar to classical elasticity. A number of these theories exist but one of the simplest is that of micropolar elasticity [1]. It is applicable to heterogeneous materials with a matrix that is stiffer than the inclusions [2].

Size effects have been observed experimentally. Structural polyurethane foam beams have displayed, in bending test, an increase in stiffness as the beam depth tends to zero [3]. Work presented in [4] describes experimental investigation of the bulk material response of a polymer MEMS cantilever beam. The results show a marked difference between the beam stiffness predicted by classical Euler-Bernoulli beam theory and the observed response. A good correlation was found to a micropolar theory based upon previous work on micropolar plates [5]. The observed beam response was one of increasing flexural stiffness with decreasing thickness.

In order to capture these size effects numerically using micropolar elasticity, additional constitutive properties need to be determined. These are in addition to the classical elastic constants, Young's modulus and Poisson's ratio. Unfortunately identifying these addition constitutive properties is more involved.

\section{Experimental Determination of Constitutive Properties}

The first experimental work carried out on micropolar materials, to determine their constitutive properties, proved to be inconclusive [5]. Indeed the difficulty arose from the inability to find a material that exhibited micropolar material behaviour to a sufficient extent, but the methods outlined in [6] were the first treatment of the micropolar theory to separate and determine the additional constitutive properties.

A more recent review of experimental methods in generalised elastic continua is presented in [7]. The experimental determination of the constitutive properties can be divided into three methods; size effect methods, field methods and wave methods.

The size effects method, used in [8], makes use of the dependency of stiffness upon size of sample. A method that is capable of determining all six micropolar constitutive 
properties by use of electromagnetic torque generation and interferometric determination of angular displacements is presented in [7]. The stiffness of circular rod specimens of decreasing size were tested in bending and torsion, and these results were compared against analytical solutions to extract the elastic constants. The electromagnetic torque generator was used so as to minimize local loading errors that can obscure size effects in smaller samples.

Field methods can be used to determine the continuum theory that the material is exhibiting. In [9] an analysis of the strain field on the surface of a rectangular section under torsion is presented. It was found that at the edge of the rectangular section the shear strain was none zero, which is not predicted in classical elasticity. A screening method was presented in [10] that used a holographic image to detect the motion of a small corner crack. This motion would be present in a micropolar material but not in a classical continuum material.

Wave methods use the propagation of stress waves to determine the constitutive properties. Micropolar materials exhibit dispersion of plane waves, although this can present difficulties as this dispersion can also be attributed to a viscoelastic response [7]. The advantage of the wave method is that they can be used for large scale materials, e.g. rock formations, which cannot be treated practically using the size effects method.

\section{Numerical Determination of Constitutive Properties}

The use of finite element modelling of the discrete micro structure to determine the micropolar in plane shear and rotational moduli of unidirectional fiber composites with fiber-matrix interfacial de-bonding is suggested in [11]. Subsequently in [12] a finite element homogenisation method to determine micropolar constitutive properties is presented in the context of composite laminates with high stress gradients.

\section{Numerical Modelling}

Computer methods, in particular the finite element method (FEM) have shown limited success in modelling micropolar elasticity. An alternative, the control volume finite element method (CVFEM), has been developed which has shown increased accuracy over the FEM [13]. This is assumed to be due to a condition of local equilibrium being imposed rather than just the global equilibrium that is enforced in the FEM.

\section{Objective}

The objective of this work is to capture an experimentally observed size effect, in a beam of a model micropolar material under a bending load, using numerical models. In order to achieve this an additional material constant is needed over and above the classical engineering constants. This material constant is gained in two ways. The first is experimentally using the size effects method [3] then secondly using a fully detailed finite element model, in ANSYS, once again using the size effect method. 


\section{Micropolar Elasticity}

Micropolar elasticity is capable of describing size effects due to the introduction of a length scale dependent coupled stress, $m$, and an additional degree of freedom, a microrotation, $\phi$. For a linear elastic isotropic micropolar material the force stress tensor and couple stress tensor respectively are,

$$
\begin{gathered}
\tau_{k l}=\lambda \varepsilon_{m m} \delta_{k l}+(2 \mu+\kappa) \varepsilon_{k l}+\kappa e_{k l m}\left(\theta_{m}-\phi_{m}\right) \\
m_{k l}=\alpha \phi_{m, m} \delta_{k l}+\beta \phi_{k, l}+\gamma \phi_{l, k}
\end{gathered}
$$

where $\tau$ is the force stress tensor, $m$ is the couple stress tensor, $\varepsilon$ is the strain tensor, $\theta$ is the macro rotation. The macro rotation is usually kinematically distinct from the microrotation but in this formulation a special case where they are equal will be used to derive a simple bending equation for a micropolar beam [14].

From the moment curvature relationship, see appendix A, the maximum displacement, $v_{\max }$ for a micropolar beam under 3 point bending is,

$$
v_{\max }=\frac{W L^{3}}{48\left(E_{m} I+\gamma A\right)}
$$

where $W(N)$ is the central applied load, $L(m)$ is the length of the beam, $E_{m}\left(\mathrm{Nm}^{-2}\right)$ is the micropolar Young's Modulus, $I\left(m^{4}\right)$ is the second moment of area, $\gamma(N)$ is a length scale dependent micropolar constant and $A\left(\mathrm{~m}^{2}\right)$ is the crossectional area. This can be rearranged to express the stiffness, $K\left(\mathrm{Nm}^{-1}\right)$ in terms of the beam depth $d$.

For a rectangular cross section the substitutions for the second moment of area, $I$, and area, $A$, are,

$$
\begin{array}{r}
I=\frac{b d^{3}}{12} \\
A=b d
\end{array}
$$

where $b$ is the breadth and $d$ is the depth, see figure 1 .

$$
K=4 E_{m} b\left(\frac{d}{L}\right)^{3}\left(1+\left[\frac{l_{r}}{d}\right]^{2}\right)
$$

where $l_{r}(m)$ is the characteristic length in bending for a rectangular cross section,

$$
l_{r}=\sqrt{\frac{12 \gamma}{E_{m}}}
$$

For a classically elastic beam the equation for determining the maximum deflection of a beam under three point bending load is

$$
v_{\max }=\frac{W L^{3}}{48 E_{m} I}
$$

therefore the stiffness is 


$$
K=4 E_{m} b\left(\frac{d}{L}\right)^{3}
$$

from this it can be seen that in equation 6 the expression outside the bracket is that of the classical beam equation and inside is the term associated with the micropolar stiffening. It can also be seen that as the depth of the beam increases the significance of the characteristic length reduces and the solution converges to the equation for a classically elastic beam.

\section{Micropolar Beam Element}

A four degree of freedom straight micropolar beam element was developed to capture the size effect numerically. The four degrees of freedom describe a cubic lateral displacement field $\left(w_{n}, \theta_{n}\right)$ ( $n=1,2$ cycling for the number of nodes). The derivation follow that of a standard classical beam element [15] but the constitutive relationship has been altered to take account of the micropolar elasticity. The stiffness matrix, $\mathbf{K}$, is

$$
\mathbf{K}=\frac{\left(E_{m} I\right)+(\gamma A)}{L^{3}}\left[\begin{array}{cccc}
12 & 6 L & -12 & 6 L \\
6 L & 4 L^{2} & -6 L & 2 L^{2} \\
-12 & -6 L & 12 & -6 L \\
6 L & 2 L^{2} & -6 L & 4 L^{2}
\end{array}\right]
$$

where the symbols have the meaning already stated.

\section{Experimental and Numerical Results}

A model material was manufactured from aluminium bar, $E=68.9 \mathrm{MNm}^{-2}, \nu=0.3$, with a regular pattern of holes, where the holes pass through the axis of bending (Figure 1). The bar was tested in 3 point bending for various beam depths, $d$ while maintaining a constant ratio of length, $L$, to depth (Figure 2). If the material were classical, the stiffness would remain constant, as the $L / d$ is constant, see equation 9 .

The hole pattern is arranged in hexagonal lattice (Figure 3) with horizontal pitch $P_{1}=0.0117 \mathrm{~m}$ and vertical pitch $P_{2}=0.0127 \mathrm{~m}$. Four test samples were made, with one hole to depth to four holes to depth (See Table 1). Once the loaded deflection data had been gained a multiple curve fit was used to gain values for $\gamma$ and $E_{m}$ from equation 6 .

The beam sample were loaded within the elastic region and stiffness results are shown in figure 3. A continuous beam of the same material was also tested and results are shown in figure 4 also. Further to this FEA analysis of the beam, modelling all the discrete detail was carried out for the model heterogeneous beams, again shown in figure 4 . There is a distinct size effect present in both the physical test and the FEA results. Carrying out a multiple parameter curve fit of the test data to find $E_{m}$ and $\gamma$ for both experiment and analysis give rise to the results shown in table 2 .

Now that the constitutive properties have been found experimentally and numeri- 
cally they can be used in numerical models. Figures 5 and 6 show the results from a micropolar beam element while figures 7 and 8 depict the results for a micropolar plane stress element. The difference between the numerical and experimental results is due to approximations in the curve fit. There is good agreement between analytical solutions and the numerical procedures. Clearly the size effect has been captured in a numerical procedure based on a micropolar continuum model. They are able to show a similar displacement field to the detailed ANSYS FEA model at a significantly reduced computational cost.

\section{Discussion}

From the experimental results (Figure 3) a few points of interest are noted. Shear deformation may be playing a role at the selected $L / d$ ratio, classical results become less stiff as the depth increases. In the micropolar plane element shear deformation is governed by an additional parameter, the coupling number, which the beam analysis ignores. However micropolar plane element results, figures 7 and 8 , indicate that predicted deformation is insensitive to coupling number, $0 \leq N \geq 1$, therefore the role of shear deformation is secondary.

The voids in the matrix material are large in comparison to the beam depth to reduce the influence of the systematic error and emphasis the size effect. Problems had been identified in past work that the micropolar behaviour is often masked but the error in the experimental procedure [3]. If the void size were sufficiently small the increase in bending stiffness could be within the systematic error of the test procedure. The disadvantage of large voids is that they increase the local loading effects, this can be seen in the micropolar test samples 2 and 3 being of similar stiffness. It is therefore suggested that there is a region in which the number of voids is large enough to average out any local loading variation but not so great that the size effect is masked by the testing procedure itself.

At present experiments to determine constitutive properties are limited to procedures where analytical solutions exist. The coupling number, found from torsion tests, could be extracted using the micropolar plane element by introducing shear into the same beam sample used in this work. Shear can be introduced by decreasing the $L / d$ ratio. From these results the 2D element could be used as a curve fitting tool to fit for the coupling number. This has the advantage over previous methods that only one sample geometry is required.

\section{Conclusion}

It has been shown that a size effect can be identified in a model heterogeneous material that can be described by micropolar elasticity. The size effect can also be identified in an FE model of the discrete detail of the material which opens the possibility of determining the correct constitutive relationships without lengthy physical testing. Furthermore analytical solutions and numerical methods for solving the micropolar beam problem 
have been developed that are able to correctly determine deflection of the micropolar beam with the size effect present.

\section{A Micropolar Beam Derivation}

Once the microrotation is no longer kinematically distinct this simplifies the formulation and the curvature, $R$ of a beam under pure bending, being bent through a small angle is,

$$
\frac{1}{R}=\frac{d \theta}{d x}=\frac{d \phi}{d x}=-\frac{d^{2} v}{d x^{2}}
$$

Considering only the out of plane couple stress, $m_{z}$ and direct stress $\sigma_{x}$

$$
\begin{gathered}
m_{z}=\gamma \frac{d \phi}{d x} \\
\sigma_{x}=\frac{E_{m} y}{R}
\end{gathered}
$$

Taking the internal resisting moment equal to any externally applied moment, $M$,

$$
M=\int_{A} y \sigma_{x}+m_{z} d A
$$

Substituting for $m_{z}=\frac{\gamma}{R}$ and $\sigma_{x}=\frac{E_{m} y}{R}$,

$$
M=\frac{1}{R} \int_{A}\left(y^{2} E_{m}+\gamma\right) d A
$$

Completing the integration where the second moment of area, $I$ is,

$$
I=\int_{A} y^{2} d A
$$

and area, $\mathrm{A}$ is

$$
A=\int_{A} d A
$$

And substituting for $\frac{1}{R}=-\frac{d^{2} v}{d x^{2}}$ the curvature relationship is,

$$
\frac{d^{2} v}{d x^{2}}=-\frac{M}{E_{m} I+\gamma A}
$$

\section{References}

[1] A. C. Eringen. Microcontinuum Field Theories I: Foundations and Solids. Springer-Verlag New York, 1999. 
[2] D. Bigoni and W. J. Drugan. Analytical derivation of cosserat moduli via homogenization of heterogeneous elastic materials. Journal of Applied Mechanics, 74(4):741-753, 2007.

[3] R.S. Lakes. Experimental microelasticity of two porous solids. International Journal of Solids and Structures, 22(1):55 - 63, 1986.

[4] Andrew W. Mcfarland and Jonathan S. Colton. Role of material microstructure in plate stiffness with relevance to microcantilever sensors. Journal of Micromechanics and Microengineering, 15(5):1060-1067, May 2005.

[5] R. D. Gauthier. Experimental investigations of micropolar media. World Scientific, Singapore, 1981.

[6] R. D. Gauthier and W. E. Jahsman. A quest for micropolar elastic constants. Journal of Applied Mechanics, 42:369-374, 1975.

[7] R. S. Lakes. Experimental methods for study of cosserat elastic solids and other generalized elastic continua. Continuum models for materials with micro-structure, 1995.

[8] R. D. Gauthier and W. E. Jahsman. Bending of a curved bar of micropolar elastic material. Journal of Applied Mechanics, 43:502-503, 1976.

[9] H. C. Park and R. S. Lakes. Torsion of a micropolar elastic prism of square crosssection. International Journal of Solids and Structures, 23(4):485-503, 1987.

[10] R. S. Lakes, D. Gorman, and W. Bonfield. Holographic screening method for microelastic solids. Journal of Materials Science, 20(8):2882-2888, 1985.

[11] Parsaoran Hutapea and Pizhong Qiao. Micropolar in-plane shear and rotation moduli of unidirectional fiber composites with fiber-matrix interfacial debonding. Journal of Composite Materials, 36(11):1381-1399, June 2002.

[12] P. Hutapea, F. G. Yuan, and N. J. Pagano. Micro-stress prediction in composite laminates with high stress gradients. International Journal of Solids and Structures, pages 2215-2248, May 2003.

[13] M. A. Wheel. A control volume-based finite element method for plane micropolar elasticity. International Journal for Numerical Methods in Engineering, 75(8):9921006, 2008.

[14] Fuang-Yuan Huang, Biing-Hwa Yan, Jyy-Liang Yan, and Der-Uei Yang. Bending analysis of micropolar elastic beam using a 3-d finite element method. International Journal of Engineering Science, 38(3):275 - 286, 2000.

[15] Robert D. Cook, David S. Malkus, and Michael E. Plesha. Concepts and Applications of Finite Element Analysis. John Wiley \& Sons, third edition, 1989. 
Table 1: Size effect test sample. Dimensions and stiffness results

\begin{tabular}{|c|c|c|c|c|}
\hline Beam & Depth $(\mathrm{mm})$ & Length $(\mathrm{mm})$ & $\mathrm{L} / \mathrm{h}$ & Stiffness $(\mathrm{N} / \mathrm{m})$ \\
\hline 1 & 12.7 & 100 & 7.8 & $4.335 \mathrm{e} 6$ \\
2 & 25.4 & 200 & 7.8 & $3.238 \mathrm{e} 6$ \\
3 & 38.1 & 300 & 7.8 & $3.242 \mathrm{e} 6$ \\
4 & 50.8 & 400 & 7.8 & $2.741 \mathrm{e} 6$ \\
\hline
\end{tabular}

Table 2: Constitutive properties from size effect experiments. Physical Test, EXP and detailed ANSYS model, ANSYS

\begin{tabular}{|c|c|c|c|}
\hline & $E_{m}\left(\mathrm{Nm}^{-2}\right)$ & $\gamma(N)$ & $l_{r}(\mathrm{~m})$ \\
\hline EXP & $2.748 \mathrm{e} 10$ & $1.923 \mathrm{e} 5$ & 0.00916 \\
ANSYS & $2.757 \mathrm{e} 10$ & $1.684 \mathrm{e} 5$ & 0.00856 \\
\hline
\end{tabular}




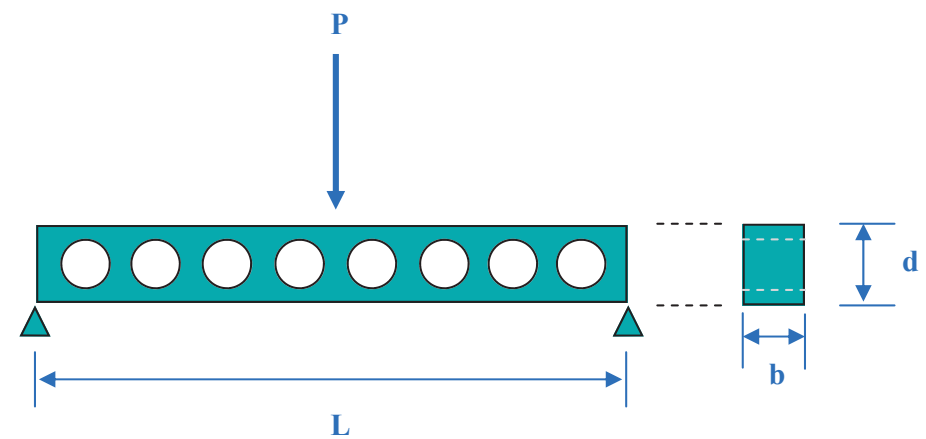

Figure 1: Heterogeneous beam in 3 point bending. Applied load P, length L, breadth, $\mathrm{b}$ and depth d

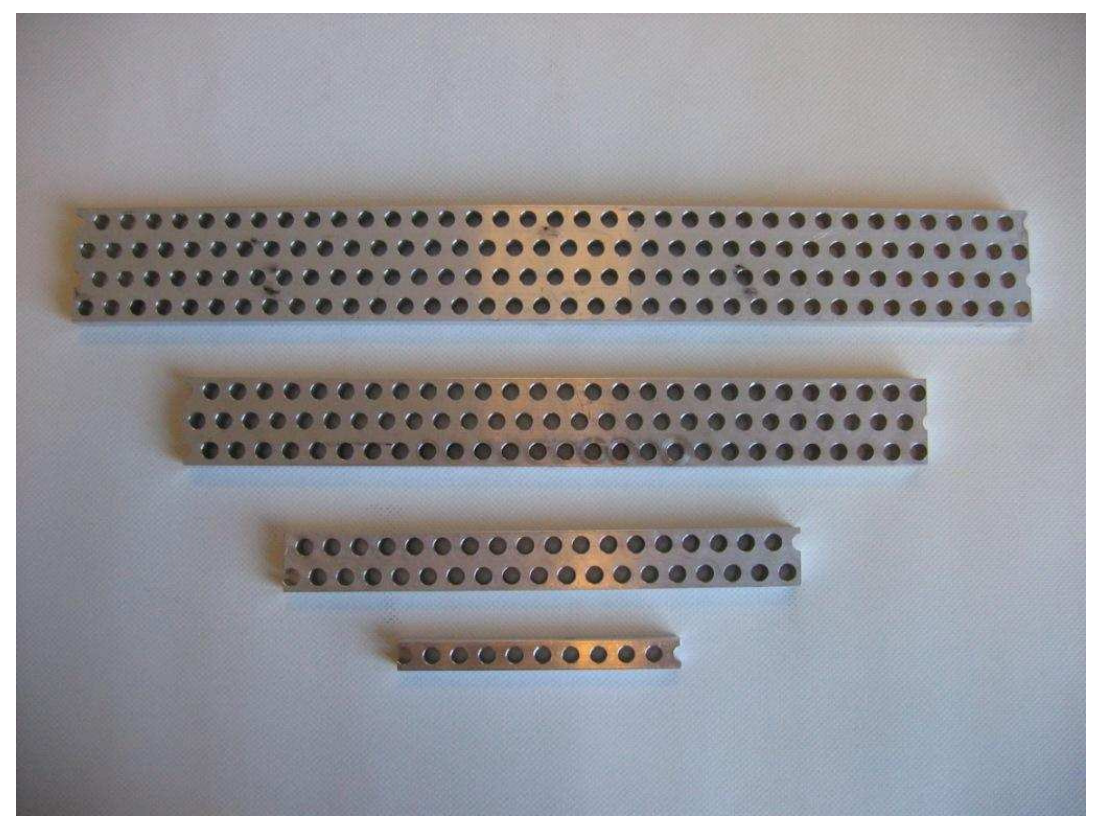

Figure 2: Model heterogeneous beam test samples, constant $L / h$ 


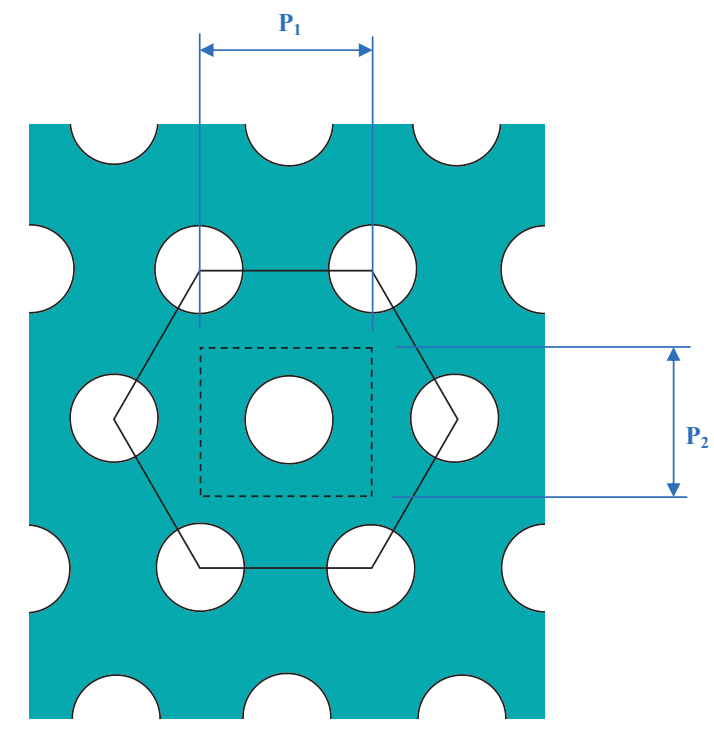

Figure 3: Hexagonal hole pattern of model heterogeneous material with horizontal pitch, $P_{1}$ and vertical pitch $P_{2}$

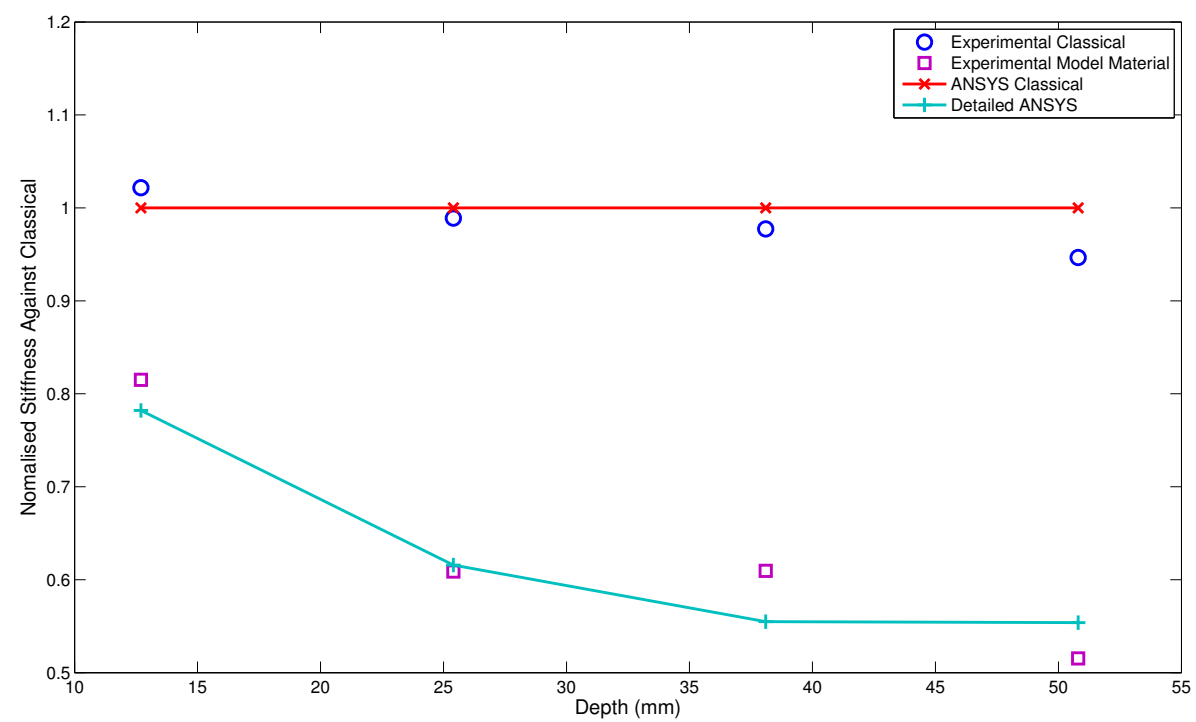

Figure 4: Experimental and detailed ANSYS results for model material 


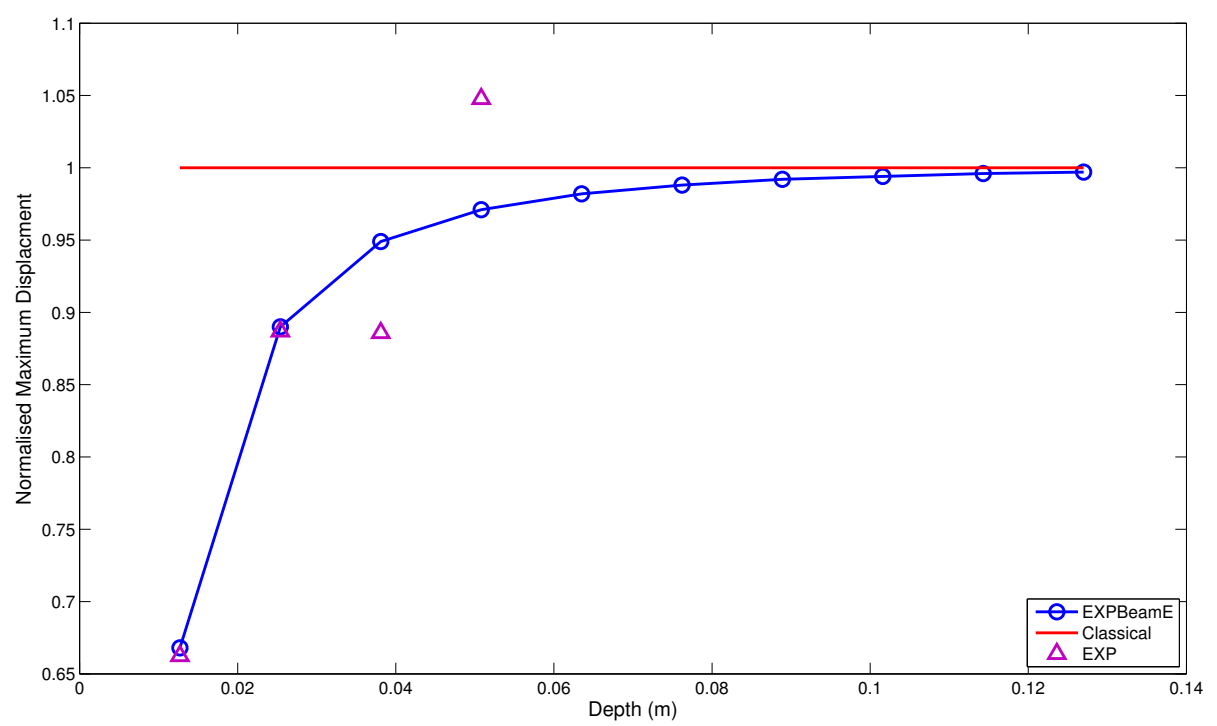

Figure 5: Maximum displacement result from Micropolar Beam element (EXPBeamE) using constitutive properties from 3 point bending test of model micropolar material, for a given load $(100 N)$. Plotted against displacements gained from experimental stiffness value $(\mathrm{EXP})$.

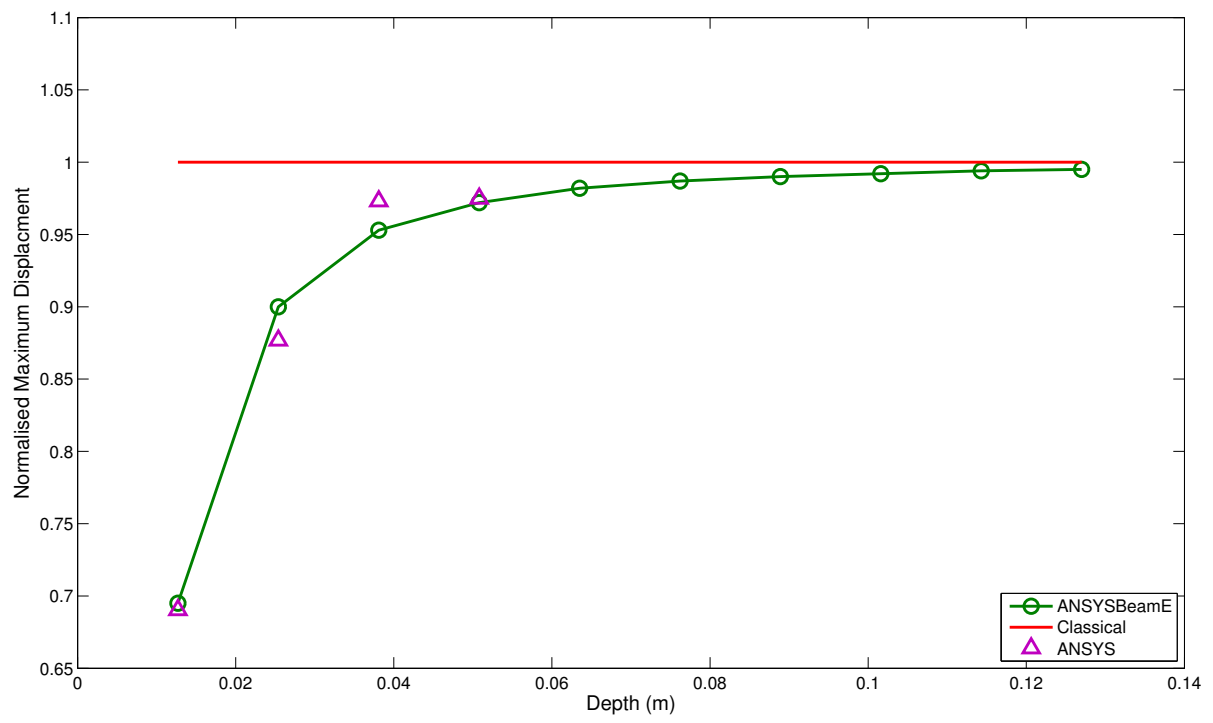

Figure 6: Maximum displacement result from Micropolar Beam element (ANSYSBeamE) using constitutive properties from detailed ANSYS model material in 3 point bending, for a given load $(100 N)$. Plotted against displacements gained from ANSYS stiffness value (ANSYS). 


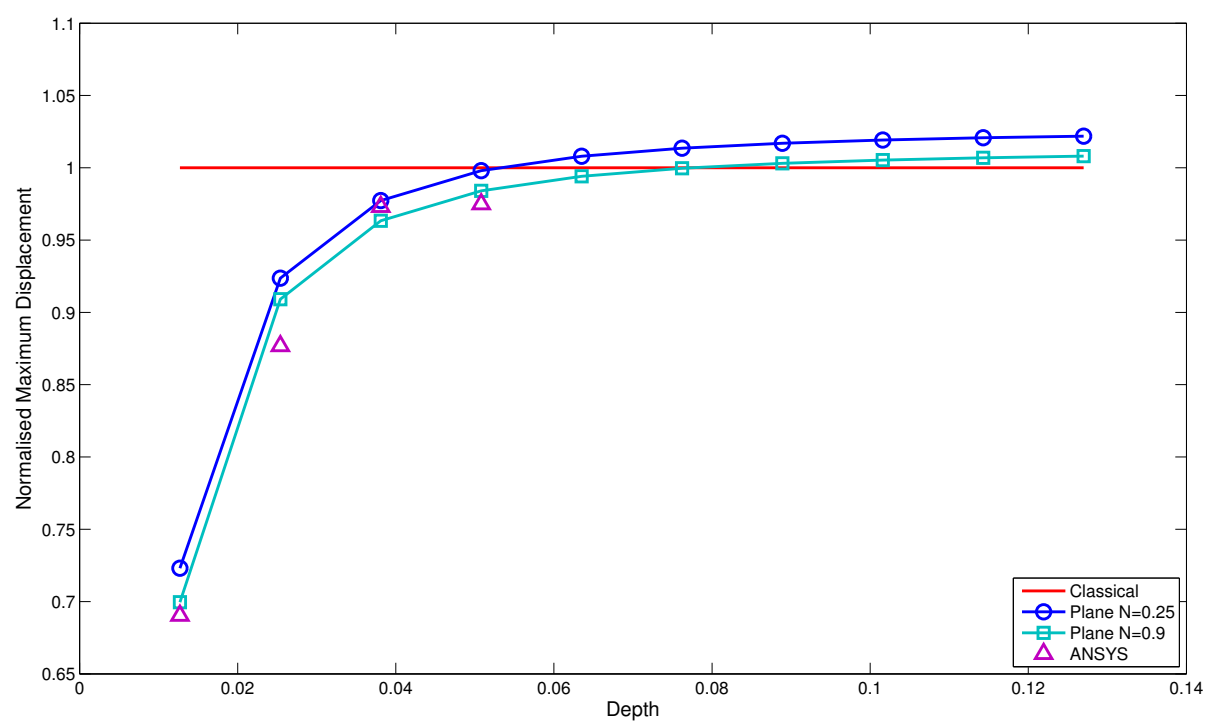

Figure 7: Maximum displacement results from micropolar plane element (Plane), for various coupling number $N$, using constitutive properties from detailed ANSYS model material in 3 point bending, for a given load $(100 N)$. Plotted against displacements gained from ANSYS stiffness value (ANSYS)

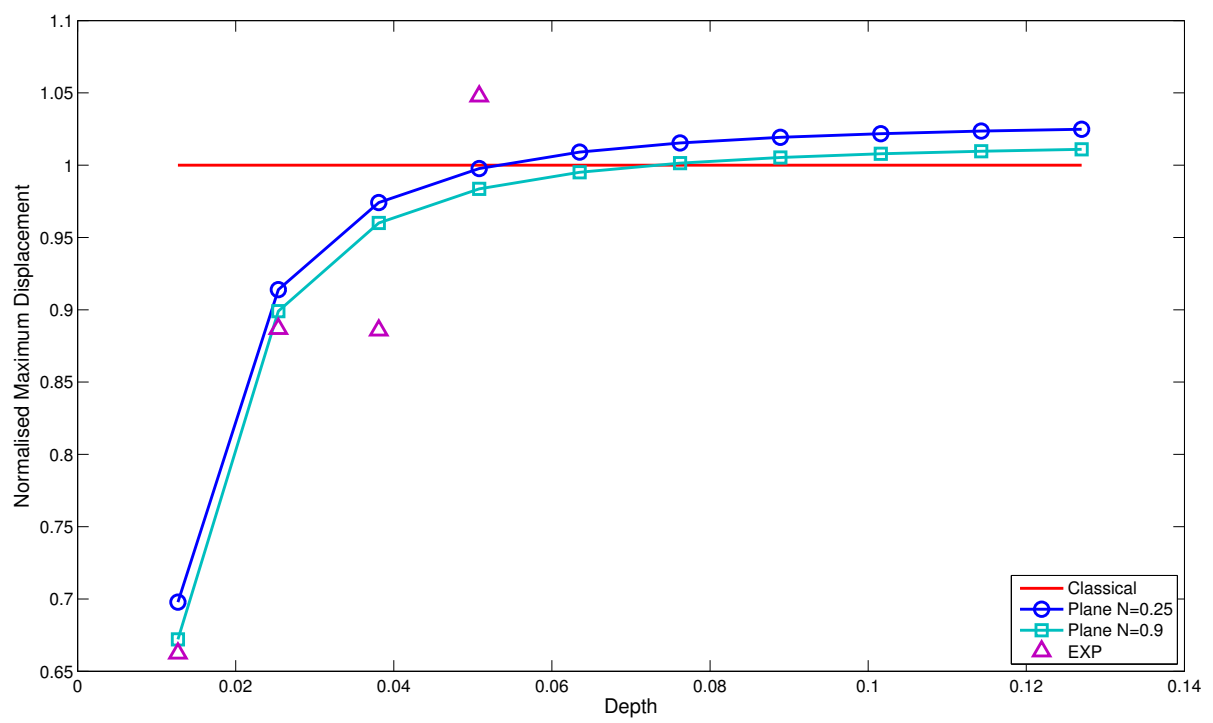

Figure 8: Maximum displacement results from micropolar plane element (Plane), for various coupling number $N$, using constitutive properties from 3 point bending test of model micropolar material, for a given load $(100 N)$. Plotted against displacements gained from experimental stiffness value (EXP). 